\title{
TNFa Induces Multidrug Resistance-Associated Protein 4 Expression through p38-E2F1-Nrf2 Signaling in Obstructive Cholestasis
}

\author{
Wei Lian, Xiaocong Liu, and Wensheng Chen \\ Department of Gastroenterology, Southwest Hospital, Army Medical University (Third Military Medical University), Chongqing, China.
}

\begin{abstract}
Purpose: To explore the molecular mechanism of the upregulation of multidrug resistance-associated protein 4 (MRP4) in cholestasis. Materials and Methods: The mRNA and protein levels of MRP4 in liver samples from cholestatic patients were determined by quantitative real-time PCR and Western blot. In human hepatoma HepG2 cells, electrophoretic mobility shift assay (EMSA) was used to determine the affinity of nuclear factor-E2-related factor (Nrf2) binding to MRP4 promoter. Dual-luciferase reporter assay was used to detect the binding of tumor necrosis factor $\alpha(\mathrm{TNF} \alpha)$ to the promotor of E2F1. The bile duct ligation mouse models were established using male C57BL/6 mice.

Results: The mRNA and protein levels of MRP4 were significantly increased in cholestatic patients. TNF $\alpha$ treatment induced the expression of MRP4 and Nrf2 and enhanced cell nuclear extract binding activity to MRP4 promoter, as demonstrated by EMSA. Nrf2 knockdown reduced MRP4 mRNA levels in both HepG2 and Hep-3B cells. In addition, TNF $\alpha$ increased Rb phosphorylation and expression of MRP4 and Nrf2 and activated E2F1 and phosphorylated p38 in HepG2 and Hep-3B cells. These effects were markedly inhibited by pretreatment with E2F1 siRNA. Dual-luciferase reporter assay validated that TNF $\alpha$ induces the transcription of E2F1. Furthermore, the expression of MRP4, Nrf2, E2F1, and p-p38 proteins was improved with treatment of TNF $\alpha$ in a mouse model of cholestasis. E2F1 siRNA lentivirus or SB 203580 (p38 inhibitor) inhibited these positive effects.

Conclusion: Our findings indicated that TNF $\alpha$ induces hepatic MRP4 expression through activation of the p38-E2F1-Nrf2 signaling pathway in human obstructive cholestasis.
\end{abstract}

Key Words: Obstructive cholestasis, multidrug resistance-associated protein 4, Nrf-2, TNF $\alpha$

\section{INTRODUCTION}

Obstructive cholestasis is defined as a mechanical blockage in the bile ducts, which prevents bile from flowing into the duodenum, leading to accumulation of potentially toxic biliary compounds in the liver and bile ducts. ${ }^{1}$ Toxicity constituents, such as

Received: March 22, 2019 Revised: July 24, 2019

Accepted: August 14, 2019

Corresponding author: Wensheng Chen, PhD, Department of Gastroenterology, Southwest Hospital, Army Medical University (Third Military Medical University), No. 30, Gaotanyan Main Street, Shapingba District, Chongqing, 400038, China. Tel: 86-023-68754124, Fax: 86-023-65415803, E-mail: wenshengchenwsc@163.com

-The authors have no potential conflicts of interest to disclose

(c) Copyright: Yonsei University College of Medicine 2019

This is an Open Access article distributed under the terms of the Creative Commons Attribution Non-Commercial License (https://creativecommons.org/licenses/ by-nc/4.0) which permits unrestricted non-commercial use, distribution, and reproduction in any medium, provided the original work is properly cited. bile acids and bilirubin, can injure hepatocytes and bile duct cells. Long-term cholestasis may develop into fibrosis, cirrhosis, and even carcinoma. The spontaneous anticholestatic defense mechanisms of hepatocytes comprise adaptive induction of basolateral alternative export pumps, which are important canalicular bile transporters. Interestingly, expression levels of hepatic membrane transporters and bile acid metabolic enzymes have been shown to be significantly altered in cholestasis. ${ }^{2-4}$ For example, as an ATP-dependent organic anion transporter belonging to the $\mathrm{ABC}$ transporter superfamily, multidrug resistance-associated protein 4 (MRP4/ABCC4) deficiency has been found to worsen liver injury and decrease plasma bile acid levels in bile duct-ligated mice. ${ }^{5-7}$ MRP4 is widely expressed in many human and rodent tissues, such as liver, kidney, intestine, brain, and breast. Published studies have shown that MRP4 expression is substantially increased in the livers of mice with disruption of bile acid nuclear receptor, accompany by increased levels of 
serum and hepatocellular bile acids, and that MRP4 can be further upregulated by cholic acid feeding, consistent with a role in bile acid homeostasis. ${ }^{8}$ Thus, hepatic MRP4 levels are normally low in rodents, but elevated in obstructive cholestasis, similar to elevated multidrug resistance-associated protein 3 (MRP3/ ABCC3) expression in obstructive cholestasis. ${ }^{9,10}$

The transcriptional regulation of hepatic organic anion transporters comprises a complex interacting network of ligand-activated nuclear receptors, including farnesoid X-activated receptor, pregnane $\mathrm{X}$ receptor (PXR), constitutive androstane receptor (CAR), retinoid X receptor, nuclear factor-E2-related factor (Nrf2), liver receptor homolog-1 (LRH-1), peroxisome proliferator-activated receptor- $\alpha$, and others. These nuclear factors play essential roles in the transcriptional regulation of hepatobiliary transport systems and have been reported to be involved in bile acid metabolism in animal models of cholestasis and cell lines. ${ }^{11,12}$ In addition, Nrf2 has been reported to participate in the transcriptional regulation of drug transporter genes, MRPs. ${ }^{13}$ The mechanisms by which proinflammatory cytokines modulate transporter gene expression have been intensively studied over the past decade. Recent reports indicate that the proposed mechanism by which tumor necrosis factor $\alpha$ (TNF $\alpha$ ) upregulates MRP3 is via induction of the liver receptor LRH-1. ${ }^{14}$ Thus, TNF $\alpha$ may be the factor that mediates bile acid-dependent induction because it upregulates LRH-1 expression in vitro.

In a previous study, we found that the upregulation of hepatic MRP3/ABCC3 expression in human obstructive cholestasis is likely triggered by TNF $\alpha$ and mediated by the activation of SP1. ${ }^{15}$ However, whether TNF $\alpha$ signaling plays a role in Nrf2-stimulated MRP4/ABCC4 expression is still unknown. Decades of study have shown that several intracellular stress signals, including mitogen-activated protein kinases, extracellular signal-regulated kinase (ERK), and c-Jun-N-terminal kinase (JNK), can be stimulated by lipopolysaccharides in hepatic Kupffer cells. ${ }^{16}$ In this study, we investigated whether p38 MAPK signaling is involved in the TNF $\alpha$-mediated regulation of MRP4. To this end, we assessed MRP4/ABCC4 expression and plasma levels of TNF $\alpha$ in the livers of patients with obstructive cholestasis by quantitative real-time PCR (qRT-PCR), Western blot, and ELISA. The expression of MRP4, Nrf2, E2F1, p-p38 MAPK and p-Rb proteins was detected by Western blot analysis. The binding activity of Nrf2 to the MRP4/ABCC4 promoter was analyzed by electrophoretic mobility shift assay (EMSA). In this report, we described the association of increased hepatic MRP4/ABCC4 expression with elevated TNF $\alpha$ levels and enhanced Nrf2 expression, showing that $\mathrm{p} 38-\mathrm{Rb}-\mathrm{E} 2 \mathrm{~F} 1$ signaling is involved in this progression.

\section{MATERIALS AND METHODS}

\section{Patients and samples}

All liver samples were collected from patients at Southwest Hospital (Chongqing, China) with informed consent from all partic- ipants, and this study was approved by the Southwest Hospital Institutional Ethics Review Board and carried out in accordance with the Declaration of Helsinki of the World Medical Association. Control liver samples were also obtained during resection of liver metastases without cholestasis ( $n=14$; three rectal metastases, four colorectal metastases, seven colonic metastases). Cholestatic liver samples $(n=14)$ were surgically resected from patients with obstructive cholestasis caused by biliary stones originating from the intrahepatic bile duct and common bile duct. Surgery was carried on these patients out within 3 days of admission due to severe symptoms of biliary obstruction and jaundice. Neither ursodeoxycholic acid (UDCA) nor other preoperative therapy was administered. Isolated liver samples were immediately cut into small pieces and stored in liquid nitrogen. The clinical characteristics of the patients are listed in Table 1. Gender was not a factor involved in altering adaptive response gene expression in cholestatic patients.

\section{Cell culture and treatment}

Human hepatoma HepG2 cells and Hep-3B cells and human embryonic kidney HEK293T cells (ATCC, Manassas, VA, USA) were maintained at $37^{\circ} \mathrm{C}$ in DMEM (Sigma Chemical Co., St. Louis, MO, USA) containing 10\% FBS, 1\% L-glutamine and $1 \%$ streptomycin (Invitrogen, San Diego, CA, USA). Before chemical treatment, cells were serum-starved overnight and then treated with the indicated dose of chemicals for designated times. For p38-Rb-E2F1 signaling inhibition experiments, HepG2 or Hep-3B cells were transfected with E2F1 siRNA (GenePharma, Shanghai, China) for $48 \mathrm{~h}$ prior to the addition of TNFa. For eliminating reactive oxygen species (ROS), cells were pre-incubated with $1 \mathrm{mM}$ N-acetyl-L-cysteine (NAC, Beyotime Biotechnology, Shanghai, China) for $30 \mathrm{~min}$ before being exposed to TNF $\alpha$.

\section{Cell transfection}

The siRNA constructs used in this study were all purchased from GenePharma, including a negative control siRNA (neg. siRNA), Nrf2 siRNA, and E2F1 siRNA. Transfection of HepG2 cells was performed using Lipofectamine ${ }^{\circledR}$ RNAiMAX Transfection Reagent (Invitrogen, Carlsbad, CA, USA) following the manufacturer's protocol. Cells were transfected with $100 \mathrm{nM}$ siRNA and were cultured for $48 \mathrm{~h}$, after which the following experiments were performed.

\section{Animals and treatments}

Male C57BL/6 mice (8-9 weeks, weighing 21-25 g) were obtained from the experimental animal center of the Field Surgery Research Institute (Daping Hospital, Chongqing, China) and underwent bile duct ligation (BDL) or sham-operation, as previously described. ${ }^{17}$ The p38 inhibitor SB 203580 (Sigma Chemical Co.) was dissolved in 3\% DMSO. Mice in the experimental group were pretreated with $30 \mathrm{~mL}$ of SB $203580(100 \mu \mathrm{M})$ or $100 \mu \mathrm{L}$ of E2F1 siRNA lentivirus. The recombinant lentivirus of E2F1 siRNA was prepared and titered to $10^{8} \mathrm{TU} / \mathrm{mL}$. TNF $\alpha$ was 
Wei Lian, et al.

Table 1. Clinical Features of Patients

\begin{tabular}{|c|c|c|c|c|c|c|c|}
\hline No. & Gender & Age (yr) & TBIL (mg/dL) & TBA ( $\mu \mathrm{mol} / \mathrm{L})$ & ALT (IU/L) & AST (IU/L) & ALP (IU/L) \\
\hline \multicolumn{8}{|l|}{ Control (n=14) } \\
\hline 1 & Female & 44 & 0.2 & 12.4 & 24.5 & 20.6 & 92.1 \\
\hline 2 & Male & 47 & 0.3 & 15.6 & 20.3 & 29.5 & 88.6 \\
\hline 3 & Female & 54 & 0.2 & 9.3 & 58.7 & 62.3 & 130.4 \\
\hline 4 & Female & 57 & 0.1 & 17.1 & 62.5 & 65.9 & 114.2 \\
\hline 5 & Male & 47 & 0.2 & 12.9 & 13.6 & 34.1 & 94.5 \\
\hline 6 & Male & 50 & 0.3 & 14.6 & 23.8 & 29.6 & 93.4 \\
\hline 7 & Female & 51 & 0.4 & 10.3 & 20.2 & 27.4 & 37.7 \\
\hline 8 & Male & 53 & 0.1 & 9.1 & 67.3 & 48.9 & 127.2 \\
\hline 9 & Female & 48 & 0.2 & 17.6 & 64.4 & 44.2 & 98.7 \\
\hline 10 & Female & 51 & 0.2 & 9.4 & 56.6 & 45.9 & 47.9 \\
\hline 11 & Male & 53 & 0.2 & 14.7 & 20.5 & 17.4 & 44.2 \\
\hline 12 & Male & 55 & 0.3 & 9.6 & 15.6 & 28.5 & 37.3 \\
\hline 13 & Female & 44 & 0.3 & 14.9 & 57.1 & 37.4 & 73.3 \\
\hline 14 & Female & 47 & 0.2 & 8.8 & 59.0 & 31.2 & 36.8 \\
\hline Mean $\pm S D$ & & $50.0 \pm 4.0$ & $0.2 \pm 0.1$ & $12.6 \pm 3.2$ & $40.3 \pm 21.6$ & $37.4 \pm 14.5$ & $79.7 \pm 33.7$ \\
\hline \multicolumn{8}{|l|}{ Obstructive $(n=14)$} \\
\hline 1 & Male & 58 & 4.0 & 221.6 & 143.5 & 325.6 & 445.3 \\
\hline 2 & Male & 59 & 2.6 & 139.3 & 176.2 & 374.1 & 493.9 \\
\hline 3 & Female & 49 & 4.2 & 148.2 & 353.3 & 561.8 & 239.1 \\
\hline 4 & Male & 60 & 4.5 & 122.4 & 119.9 & 251.6 & 308.7 \\
\hline 5 & Female & 62 & 3.2 & 240.5 & 159.8 & 287.3 & 276.5 \\
\hline 6 & Female & 59 & 3.1 & 143.9 & 177.2 & 296.3 & 456.4 \\
\hline 7 & Male & 45 & 4.5 & 152.7 & 265.6 & 403.6 & 522.8 \\
\hline 8 & Female & 57 & 3.0 & 214.5 & 146.4 & 247.5 & 318.9 \\
\hline 9 & Female & 49 & 4.7 & 219.1 & 237.1 & 316.9 & 426.3 \\
\hline 10 & Male & 52 & 3.1 & 154.5 & 121.6 & 268.2 & 338.3 \\
\hline 11 & Male & 48 & 5.0 & 229.3 & 169.9 & 306.7 & 256.8 \\
\hline 12 & Male & 56 & 3.0 & 238.6 & 251.1 & 413.4 & 370.8 \\
\hline 13 & Female & 44 & 2.9 & 213.5 & 302.9 & 389.1 & 561.5 \\
\hline 14 & Female & 58 & 3.3 & 152.4 & 137.8 & 231.0 & 344.6 \\
\hline Mean $\pm S D$ & & $54.0 \pm 6.0$ & $3.7 \pm 0.8$ & $185.0 \pm 43.1$ & $197.3 \pm 72.5$ & $333.8 \pm 88.4$ & $382.9 \pm 102.3$ \\
\hline$p$ value (vs. control) & & 0.052 & $<0.001$ & $<0.001$ & $<0.001$ & $<0.001$ & $<0.001$ \\
\hline
\end{tabular}

TBIL, total bilirubin; TBA, total bile salts; ALT, alanine aminotransferase; AST, aspartate transaminase; ALP, alkaline phosphatase.

intravenously administered at various time periods as indicated.

\section{ELISA}

ELISA kits for human and mouse TNF $\alpha$, IL-6, and IL-1 $\beta$ detection were all purchased from Beyotime Biotechnology. Plasma levels of TNF $\alpha$, IL-6, and IL- $1 \beta$ in cholestatic patients or in mouse models of cholestasis were assayed using the corresponding ELISA kit according to the manufacturer's instructions.

\section{Liver function}

At the end of the treatment period, blood samples were collected from the orbital veins of mice under anesthesia. After $2 \mathrm{~h}$ of coagulation at room temperature, blood samples were centrifuged at $5000 \mathrm{rpm}$ for $15 \mathrm{~min}$ The serum was collected for the detection of alanine aminotransferase (ALT) and aspartate trans- aminase (AST) levels using an automatic biochemical analyzer (AU5800 Series, Beckman Coulter, Brea, CA, USA).

\section{Detection of reactive oxygen species}

Intracellular ROS levels were determined using Reactive Oxygen Species Assay Kits (Nanjing Jiancheng Bioengineering Institute, Nanjing, China). Briefly, HepG2 cells and Hep-3B cells were cultured in DMEM in the presence of $50 \mathrm{ng} / \mathrm{mL}$ of TNF $\alpha$. After $12 \mathrm{~h}$, cells were digested with $0.25 \%$ trypsin and were then collected into centrifuge tubes. The collected cells were rinsed twice with $10 \mathrm{mM}$ fresh phosphate buffer saline, re-suspended in serum-free medium containing $10 \mu \mathrm{M}$ 2,7' -dichlorofluorescin diacetate (DCFH-DA), and then incubated in the dark for 30 $\min$ at $37^{\circ} \mathrm{C}$. At the end of incubation, cells were washed three times with serum-free medium to fully remove DCFH-DA that 
did not enter cells. The intensity of ROS fluorescence was measured by flow cytometry (BD Biosciences, San Diego, CA, USA) at the wavelength of $488 \mathrm{~nm}$.

\section{RNA extraction and qRT-PCR}

Samples of liver tissue frozen ( $100 \mathrm{mg}$ ) in liquid nitrogen were ground and subsequently used for the extraction of total RNA with TRIzol reagent (Invitrogen). Total RNA was reverse transcribed into cDNA using a Revert Aid ${ }^{\mathrm{TM}}$ first strand cDNA synthesis kit (MBI Fermentas Inc., Ontario, Canada). qRT-PCR using a SYBR ${ }^{\circledR}$ premix Ex Taq ${ }^{\mathrm{TM}}$ II kit (Takara Biotechnology, Tokyo, Japan) was performed in a Bio-Rad CFX96 real-time system machine (Bio-Rad, Hercules, CA, USA) to determine the mRNA levels of specific genes, whose primers are listed in Table 2. The amplification efficiency of target genes was considered using CFX manager 2.0 (Bio-Rad) for data analysis. GAPDH was used as a reference for normalizing data.

\section{Western blot analysis}

Total membranes and nuclear extracts were prepared as described previously, ${ }^{18}$ and protein concentrations were determined using a Bradford kit (Thermo Scientific, Rockford, IL, USA). The dilutions of primary antibodies were MRP4, 1:1300; Nrf2, 1:1000; p-p38 MAPK, 1:2000; E2F1, 1:2000; and p-Rb, 1:2500 (Cell Signaling, Danvers, MA, USA). GAPDH and SH-PTP1 (Santa Cruz Biotechnology, Santa Cruz, CA, USA) were used as loading references for data analysis.

\section{Electrophoretic mobility shift assay}

Oligos containing the Nrf2 response elements in the human MRP4/ABCC4 promoter or its mutant forms, as previously reported, ${ }^{18}$ were labeled with $\gamma$-32P-ATP $(10 \mathrm{mCi} / \mathrm{mL})$. EMSA was performed using nuclear extracts from HepG2 cells as described previously. ${ }^{19}$

\section{Dual-luciferase reporter assay}

cDNA fragments containing the predicted TNF $\alpha$ binding sequences in E2F1 were amplified and subcloned into the pGL3 luciferase promoter-reporter vector (Promega, Madison, WI, USA). As a control, the pGL3-mutE2F1 plasmids were also constructed using cDNA fragments containing corresponding mutated nucleotides for TNF $\alpha$. HEK-293T cells were seeded into six-well plates at $1 \times 10^{5}$ cells per well, transfected with $0.1 \mu \mathrm{g}$ of pGL3-E2F1 or pGL3-mutE2F1 vectors in the presence of preTNF $\alpha$ or pre-DMSO negative controls using Lipofectamine 2000 (Invitrogen, Carlsbad, CA, USA), and incubated for $48 \mathrm{~h}$. Cells were harvested and luciferase activities were measured using

Table 2. Sense and Antisense Primers Used for qRT-PCR

\begin{tabular}{lcl}
\hline \multicolumn{1}{c}{ Gene } & Sense primer $\left(\mathbf{5}^{\prime} \rightarrow \mathbf{3}^{\prime}\right)$ & Antisense primer $\left(\mathbf{5}^{\prime} \rightarrow \mathbf{3}^{\prime}\right)$ \\
\hline MRP4 (ABCC4) & TGCCTTTGGTCCCGATTC & TGGTGGTGGGCGTTCTGAT \\
Nrf2 & TCTATGTGCCTCCAAAGG & CTCAGCATGATGGACTTGGA \\
GAPDH & ACCACAGTCCATGCCATCAC & TCCACCACCCTGTTGCTGTA
\end{tabular}

the dual-luciferase reporter assay kit (Promega) according to the operating instructions.

\section{Statistical analysis}

All data are expressed as the mean $\pm \mathrm{SD}$ of at least three determinations and were analyzed using one-way ANOVA and subsequent Tukey's multiple comparison test for comparison of two groups. A difference was accepted as significant if $p<0.05$.

\section{RESULTS}

Hepatic MRP4 expression and plasma TNF $\alpha$ are elevated in patients with obstructive cholestasis

In this study, Western blot analysis showed that the expression of MRP4 in liver samples of patients with obstructive cholestasis was significantly increased (3.8-fold) when compared with control liver tissues samples. qRT-PCR assay revealed that the expression of MRP4 mRNA was increased 3.4-fold in liver samples of patients with obstructive cholestasis (Fig. 1A). ELISA kit
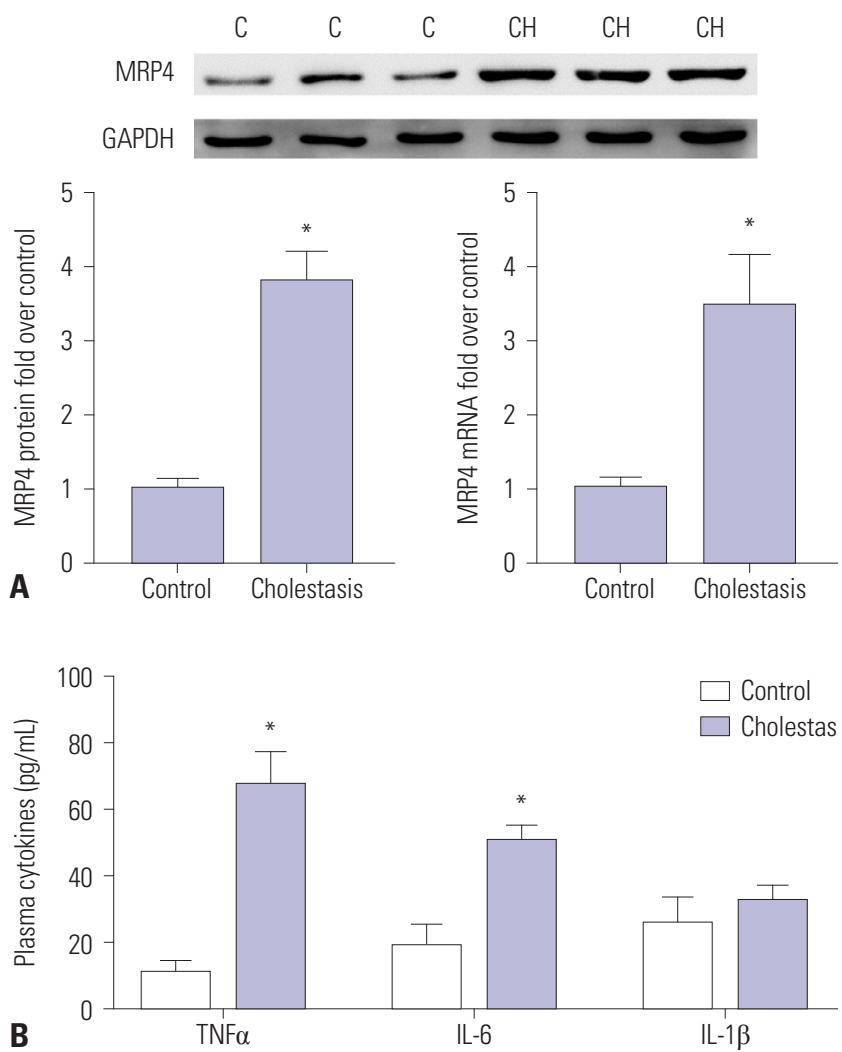

Fig. 1. Expression of MRP4 and plasma levels of TNF $\alpha$ were increased in liver samples of patients with obstructive cholestasis. (A) The expression of MRP4 protein and mRNA was detected by Western blot and qRT-PCR analysis. Representative blots and corresponding densitometry are shown ( $n=14)$. (B) Plasma levels of TNF $\alpha$, IL-6, and IL-1 $\beta$ in patients with obstructive cholestasis were detected using ELISA kits $(n=14)$. ${ }^{*} p<0.05$, vs. controls. C, control liver tissues samples; $\mathrm{CH}$, cholestasis liver samples, MRP4, multidrug resistance-associated protein 4; Nrf2, nuclear factor-E2-related factor; TNF $\alpha$, tumor necrosis factor $\alpha$. 
was used to evaluate the plasma levels of cytokines. Compared to the control group, the levels of TNF $\alpha$ and IL- 6 were significantly increased in cholestatic patients, whereas levels of IL-1 $\beta$ were not significantly altered (Fig. 1B).

\section{TNF $\alpha$ increases Nrf2 expression and DNA binding to MRP4 promoter}

Here, we determined the regulatory mechanism of the promoter of the second rescue transporter MRP4 and related receptors in response to TNF $\alpha$ in obstructive cholestasis. Our results indicated that TNF $\alpha$ increases Nrf2 mRNA and nuclear protein expression in a dose and time-dependent manner in both HepG2 (Fig. 2A) and Hep-3B cells (Fig. 2B). Furthermore, EMSA demonstrated that TNF $\alpha$ increases binding capacity to MRP4 promoter in a time-dependent manner when using the Nrf2 response element in nuclear extracts from HepG2 cells (Fig. 2C).
To confirm that Nrf2 is critical to inducing MRP4 expression, siRNA against Nrf2 was transfected into HepG2 cells or Hep-3B cells in the presence of TNF $\alpha$ treatment. As shown in Fig. 2D, the transcription of MRP4 was significantly inhibited by Nrf2-siRNA transfection. These results confirm that TNF $\alpha$ treatment induces Nrf2 expression and enhances Nrf2 binding to MRP4 promoter.

Since it has been reported that cholestasis can induce ROS production in hepatocytes and that TNF $\alpha$ increases ROS levels, we investigated whether ROS is an initial trigger that activates MRP4 under the stimulation of TNF $\alpha$. NAC, a ROS scavenger, was used to inhibit ROS production in this study. We demonstrated that ROS levels were significantly elevated in both HepG2 cells and Hep-3B cells after TNFa stimulation and were positively correlated with mRNA levels of MRP4 (Fig. 2E and F). NAC pre-treatment significantly inhibited ROS production, but did not change the mRNA levels of MRP4, compared to the TNFa
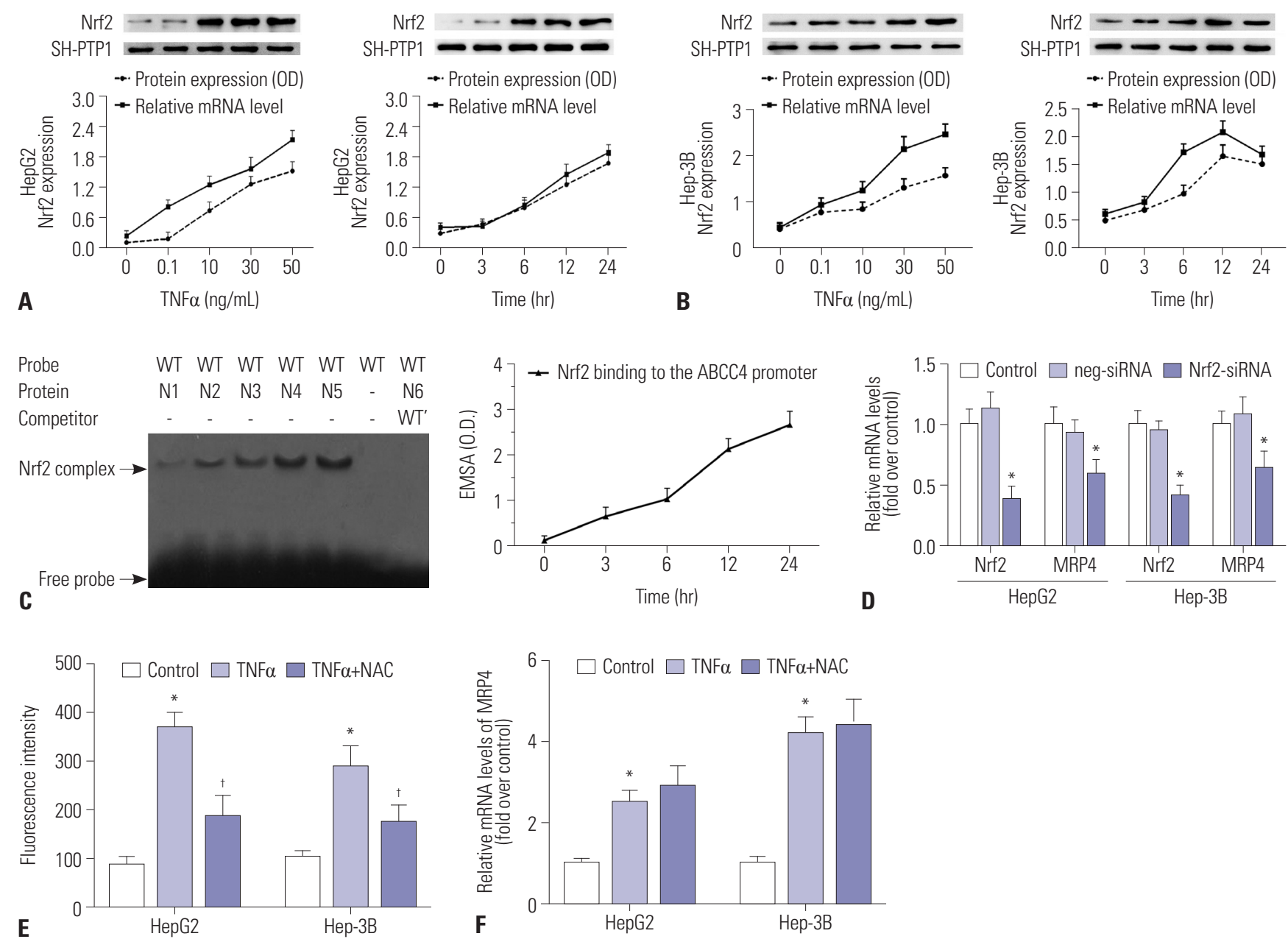

Fig. 2. TNF $\alpha$-induced Nrf2 expression and binding activity to Nrf2 response element in MRP4 promoter. The influence of TNF $\alpha$ on Nrf2 expression in HepG2 cells (A) and Hep-3B cells (B) was detected by qRT-PCR and Western blot analysis. (C) HepG2 cells were serum-starved overnight before TNFa treatment. Nrf2 binding activity to the Nrf2 response element in MRP4/ABCC4 promoter after TNF $\alpha$ treatment was detected by EMSA analysis. (D) HepG2 cells and Hep-3B cells were transfected with Nrf2 siRNA upon treatment with $50 \mathrm{ng} / \mathrm{mL}$ of TNF $\alpha$. Transcription of Nrf2 and MRP4 in HepG2 and Hep-3B cells was detected by qRT-PCR. (E) ROS levels in cells were determined using Reactive Oxygen Species Assay kits. NAC was used to eliminate ROS. The intensity of ROS fluorescence was measured by flow cytometry at $488 \mathrm{~nm}$. (F) The mRNA levels of MRP4 were detected by qRT-PCR. ${ }^{*} p<0.05$, vs. controls; ${ }^{\dagger} p<0.05$, vs. TNF $\alpha$. MRP4, multidrug resistance-associated protein 4; Nrf2, nuclear factor-E2-related factor; TNF $\alpha$, tumor necrosis factor $\alpha$; ROS, reactive oxygen species. 
group (Fig. 2E and F). These data implied that ROS is not an essential mediator of MRP4 expression stimulated by TNF $\alpha$.

\section{TNF $\alpha$ mediates MRP4/Nrf2 interactions via p38-Rb- E2F1 activation}

To investigate whether p38 MAPK and E2F1 are part of the TNF $\alpha$ enhanced MRP4/Nrf2 signaling pathway, we treated HepG2 cells and Hep-3B cells with $50 \mathrm{ng} / \mathrm{mL}$ of TNF $\alpha$ for $12 \mathrm{~h}$. The results showed that phosphorylated p38 and E2F1 were activated in a time-dependent manner of TNF $\alpha$ treatment in both HepG2 cells and Hep-3B cells (Fig. 3A). The results of luciferase reporter gene assays showed that pre-TNF $\alpha$ significantly induces luciferase activity in pGL3-E2F1-3'-UTR-transfected cells, whereas luciferase activity is not affected by pre-TNF $\alpha$ in the pGL3mutated E2F1-3'-UTR transfected group in comparison with preTNF $\alpha$-negative control treatment (Fig. 3B). When HepG2 or Hep-3B cells were pretreated with E2F1 siRNA, TNF $\alpha$-induced E2F1 activation and phosphorylation of $\mathrm{Rb}$ and p38 were abolished, whereas reductions in Nrf2 and MRP4 protein levels were observed in these cells (Fig. 3C and D).
TNF $\alpha$ injection increases MRP4/Nrf2 via p38-Rb-E2F1 pathway in mouse models of cholestasis

Expression of MRP4 protein in patients with obstructive cholestasis has been observed. In addition, we determined the expression of Nrf2 and MRP4 in BDL mice. As in patients with cholestasis, TNF $\alpha$ levels in mouse models of cholestasis were markedly upregulated, compared with levels of IL-6 and IL-1 $\beta$ (Fig. 4A). Serum levels of ALT and AST in BDL mice were also significantly elevated (Fig. 4B). The expression of MRP4 and Nrf2 proteins in Western blot analysis was induced in BDL mice when compared with sham rats. Moreover, after injection with $50 \mathrm{ng} / \mathrm{mL}$ of TNF $\alpha$, the expressions of MRP4 and Nrf2 were significantly upregulated in BDL mice (Fig. 4C). In addition, p-p38 and E2F1 expression was significantly elevated after injection with TNF $\alpha$ (Fig. 4D). Similar to results in patients with obstructive cholestasis, these data confirmed that TNF $\alpha$ participates in the regulation of MRP4/Nrf2 expression and that E2F1 may be involved in this regulatory mechanism. The expression of these proteins was decreased by pre-treatment with either E2F1 siRNA lentivirus or SB 203580.
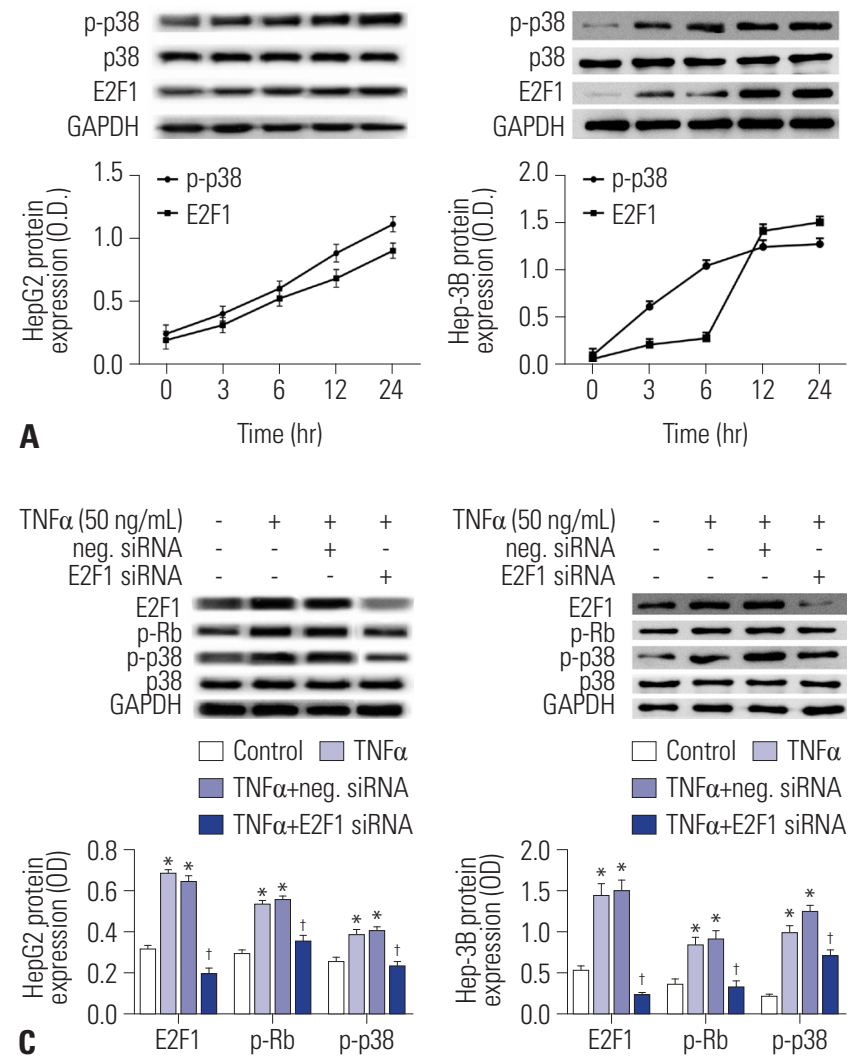
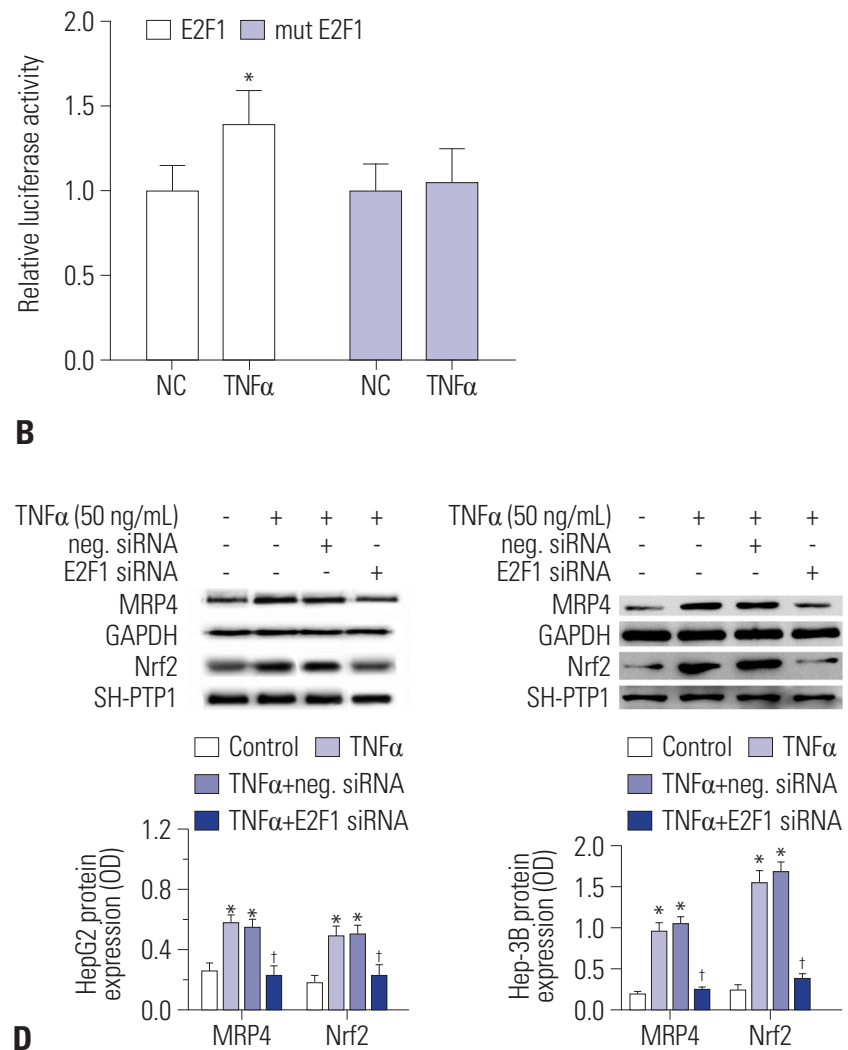

Fig. 3. The p38-Rb-E2F1 pathway mediates TNF $\alpha$-induced MRP4 expression. (A) The influence of TNF $\alpha$ on p-p38 and E2F1 in HepG2 cells and Hep-3B cells was detected by Western blot analysis. (B) The interaction between E2F1 3'-UTR and TNF $\alpha$ was detected by luciferase activity assay. Wild-type or mutated E2F1 3'-UTR containing binding sites for TNF $\alpha$ was co-transfected with pre-TNF $\alpha$ or pre-DMSO negative control in HEK293T cells. (C and D) The expression of p-p38, p38, E2F1, p-Rb, MRP4, and Nrf2 protein levels in both HepG2 cells and Hep-3B cells was detected by Western blot and their corresponding densitometry values after TNF $\alpha$ and siRNA treatment were quantified. ${ }^{*} p<0.05$, vs. controls; ${ }^{\dagger} p<0.05$, vs. TNF $\alpha$. MRP4, multidrug resistance-associated protein 4; Nrf2, nuclear factor-E2-related factor; TNF $\alpha$, tumor necrosis factor $\alpha$. 

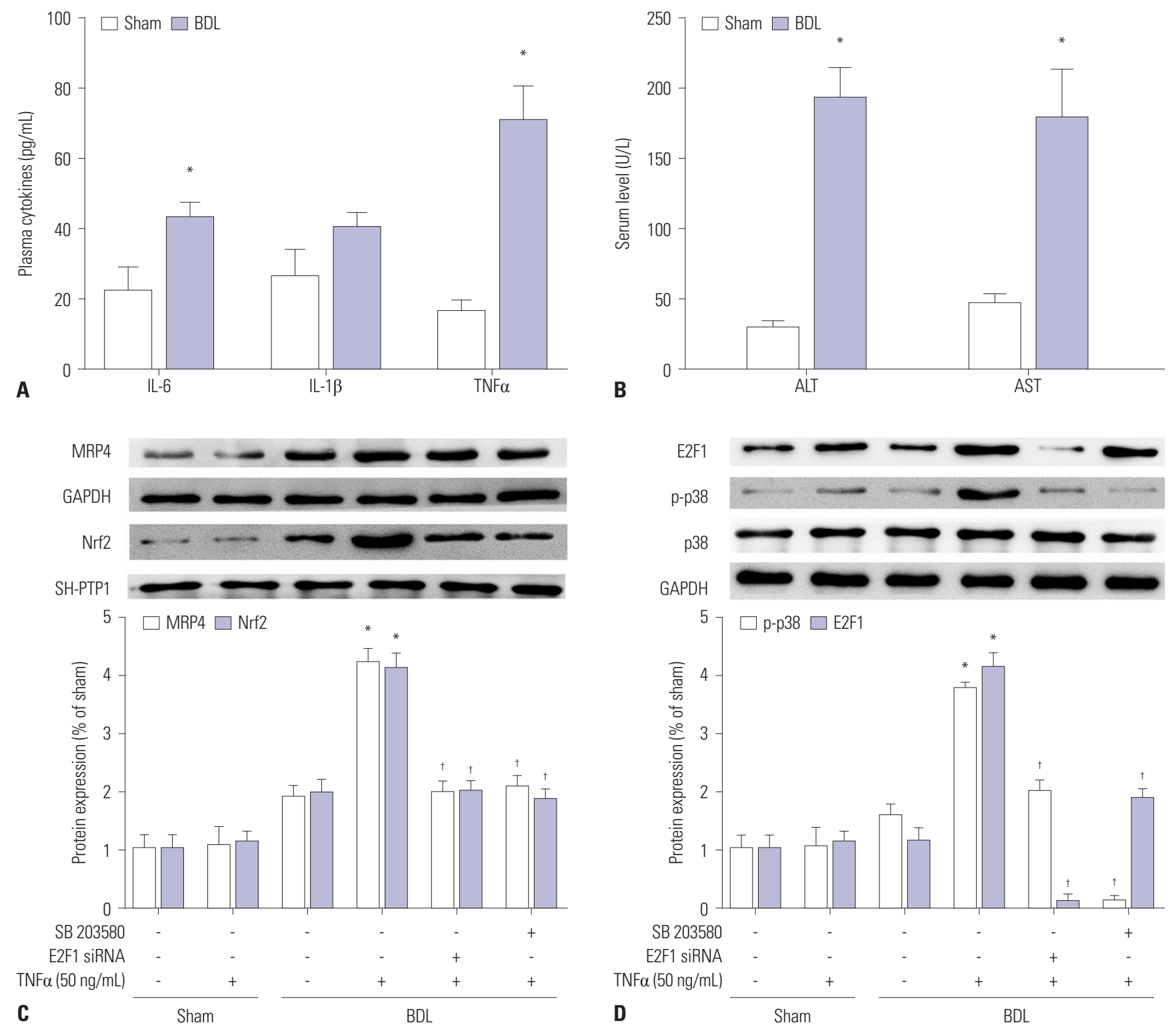

Fig. 4. The p38-Rb-E2F1 pathway mediates TNF $\alpha$-induced MRP4/ABCC4 expression in mouse models of cholestasis. (A) Plasma levels of TNF $\alpha$, IL-6, and IL-1 $\beta$ in BDL mice were detected by ELISA after BDL operation. (B) Serum levels of ALT and AST in mice were detected using an automatic biochemical meter. (C and D) Representative Western blots for MRP4, Nrf2, p-p38, and E2F1 and their corresponding densitometry values after E2F1 siRNA lentiviral, SB 203580 pretreatment and TNF $\alpha$ injection (\% of control group). ${ }^{*} p<0.05$, vs. controls. ${ }^{\dagger} p<0.05$, vs. TNF $\alpha$. MRP4, multidrug resistance-associated protein 4 ; Nrf2, nuclear factor-E2-related factor; TNF $\alpha$, tumor necrosis factor $\alpha$; ALT, alanine aminotransferase; AST, aspartate transaminase; BDL, bile duct ligation.

\section{DISCUSSION}

Cholestasis is a clinical syndrome characterized by systemic and intrahepatic retention of excessive toxic bile acids that causes liver injury, which may result either from a functional defect in bile formation at the level of the hepatocyte or from an impairment in bile secretion and flow at the bile duct level..$^{10}$ Both congenital and acquired pathogenic factors can cause bile acid secretion disorders and homeostatic imbalance, and eventually develop into cholestasis. However, treatment methods available for cholestasis and the current knowledge of related molecular mechanisms are seriously lacking. Recent research has shown that reduced expression and function of transport systems may underlie the pathogenesis of cholestasis.

In recent years, transcription factors have been proven to play crucial roles in bile acid detoxification and output in cholestasis, such as multidrug resistance-associated protein (MRP), bile salt export pump, and the basolateral $\mathrm{Na}^{+}$-dependent bile acid uptake system (NTCP) ${ }^{15,20}$ The elevated expression of MRP4/ABCC4 has been previously reported in the liver tissues of cholestasis rodent models and some patients with primary biliary cirrhosis and obstructive cholestasis. ${ }^{21}$ Upregulation of MRP4 protects against the accumulation of toxic bile acids during cholestasis by facilitating their efflux into the blood for ultimate renal excretion. ${ }^{22}$ In this report, we found that protein and mRNA expression of MRP4/ABCC4 and TNF $\alpha$ levels were significantly increased 
both in obstructive cholestatic patients and in mouse models of cholestasis. However, whether TNF $\alpha$ induction of MRP4/ABCC4 may also be mediated through an Nrf2 pathway needs further study. Thus, we explored the role of TNF $\alpha$ in hepatic MRP4 expression and the expression of related nuclear receptors, Nrf2, in the HepG2 cells, which may have major clinical and therapeutic implications.

$\mathrm{TNF} \alpha$ is a pleiotropic cytokine that mediates inflammatory, proliferative, cytostatic, and cytotoxic effects in a variety of cell types. TNF $\alpha$ has been thought to be crucial for liver injury and subsequent liver fibrosis. ${ }^{23}$ Interestingly, in contrast to its negative regulatory effects, TNF $\alpha$ plays a protective role against liver injury. ${ }^{24}$ The current study showed that proinflammatory cytokines (IL-1 $\beta$ and TNF $\alpha$ ) regulate ABCG2 and PXR expression, as well as NF- $\kappa \mathrm{B}$ activity, in some breast cancer and normal cells. ${ }^{25}$ Moreover, recent studies have shown that Nrf2 activators, such as UDCA and oltipraz, induce MRP efflux transporters in rodent liver. ${ }^{26}$ Our data demonstrated that TNF $\alpha$ significantly increases the expression of Nrf2 and enhances Nrf2 binding to the MRP4 promoter in HepG2 cells in a time- and dose-dependent manner.

Our previous study indicated that upregulation of hepatic MRP3/ABCC3 expression in human obstructive cholestasis is likely triggered by TNF $\alpha$ and mediated by activation of the JNK/ SAPK signaling pathway. ${ }^{15}$ Major members of the SAPK family include ERK, JNK, and p38 MAPK. To investigate whether p38 MAPK was part of the mechanism of TNF $\alpha$-enhanced MRP4/ Nrf2, we pretreated BDL mice with the p38 inhibitor SB 203580. Blocking p38 activity with SB 203580 had no effect on JNK, ERK, or several other protein kinases. The $\mathrm{Rb} / \mathrm{E} 2 \mathrm{~F} 1$ pathway, which is a vital regulator of G1-S phase transition, has been shown to act as a converging point for multiple signaling events. ${ }^{27}$ In Jurkat cells, $\mathrm{Rb} / \mathrm{E} 2 \mathrm{~F} 1$ is modulated by $\mathrm{p} 38$ in response to Fas activation. ${ }^{28}$ Our results showed that TNF $\alpha$ increases p-p38 and E2F1 expression. However, both E2F1 siRNA and SB 203580 blocked TNF $\alpha$ induced $\mathrm{Rb}$ phosphorylation, induction of expression of the transcription factor Nrf2, and MRP4/ABCC4, p-p38, and E2F1 expression in HepG2 cells. In the present report, we demonstrated that, indeed, $\mathrm{Rb} / \mathrm{E} 2 \mathrm{~F} 1$ is activated by TNF $\alpha$-activated p-p38. Otherwise, $\mathrm{Rb} / \mathrm{E} 2 \mathrm{~F} 1$ can modulate upstream p38 signals. Accordingly, we speculated that TNF $\alpha$-induced hepatic MRP4/ ABCC4 expression via activation of the p38-Rb-E2F1 signaling pathway is accompanied by an increase in Nrf2 expression and functions in human obstructive cholestasis.

In severe cholestasis, liver inflammation may increase $\mathrm{TNF} \alpha$ expression and result in upregulation of MRP4/ABCC4 expression. Hepatic MRP4/ABCC4 expression does not change, because TNF $\alpha$ levels may be normal in the early stages of cholestasis. Our findings indicated that Nrf2 plays a role in $\mathrm{TNF} \alpha$-mediated induction of MRP4/ABCC4 expression. Nrf2 has also been implicated in the upregulation of MRP3 in mouse liver and may theoretically be activated in obstructive cholestasis via the accumulation of hydrophobic bile acids. The mechanism of MRP4 and 3 induction has not yet been fully elucidated. Hypothetical
$\mathrm{TNF}-\alpha \longrightarrow \mathrm{p38} / \mathrm{Rb} \longrightarrow \mathrm{E} 2 \mathrm{~F} 1 \longrightarrow \mathrm{Nrf2} \longrightarrow \mathrm{MRP4}$

Fig. 5. Pathway of TNF $\alpha$-induced expression of MRP4. MRP4, multidrug resistance-associated protein 4; Nrf2, nuclear factor-E2-related factor; TNF $\alpha$, tumor necrosis factor $\alpha$.

mechanisms include upregulation via nuclear factors, such as Nrf2, which have been shown to be activated by biliary compounds. In a previous study, we demonstrated that PXR and CAR protein levels were also significantly increased in obstructive cholestasis. ${ }^{18}$ Whyte-Allman, et al. ${ }^{29}$ reported that MRP4 mRNA and protein expression was significantly upregulated in an in vitro cell line model of the blood-testis barrier after exposure to PXR or CAR ligands. Meanwhile, PXR or CAR knockdown attenuated the expression of MRP4..$^{29}$ This positive correlation between MRP4 and PXR/CAR suggested that PXR/CAR might also be involved in the regulation of $\mathrm{TNF} \alpha$-induced expression of MRP4. Nevertheless, the relative contribution of each transcription factor remains to be determined.

This study mainly focused on the mechanisms of the adaptive response of TNF $\alpha$-upregulated expression of MRP4/ABCC4 in obstructive cholestatic patients. Nevertheless, we also noted alterations in the expression of other genes (MDR3/ABCB3, NTCP/ SLC10A1) involved in bile salt transport and synthesis in obstructive cholestatic patients. These changes in gene expression were consistent with previous reports and appeared to contribute to the overall adaptive protective responses in cholestatic liver injury. ${ }^{9,30}$ A sketch map was provided in Fig. 5 to illustrate the pathway of TNF $\alpha$-induced expression of MRP4.

In summary, the current study demonstrated that the upregulation of MRP4/ABCC4 in obstructive cholestasis is positively associated with serum levels of TNF $\alpha$. We also showed that TNF $\alpha$ induces MRP4/ABCC4 expression via p38-Rb-E2F1 activation with a concomitant enhancement of Nrf2 expression in human hepatocytes. These findings suggest that the proinflammatory cytokine TNF $\alpha$ plays a cytoprotective role in cholestatic liver injury.

\section{AUTHOR CONTRIBUTIONS}

Conceptualization: Wensheng Chen. Data curation: Wei Lian. Formal analysis: Wei Lian and Xiaocong Liu. Methodology: Wei Lian and Xiaocong Liu. Project administration: Wensheng Chen. Resources: Wei Lian and Xiaocong Liu. Software: Xiaocong Liu. Supervision: Wensheng Chen. Validation: Wensheng Chen. Visualization: Wei Lian. Writing—original draft: Wei Lian. Writing—review \& editing: Wensheng Chen, Wei Lian, and Xiaocong Liu.

\section{ORCID iDs}

Wei Lian https://orcid.org/0000-0001-7006-4315

Xiaocong Liu https://orcid.org/0000-0003-1760-8162

Wensheng Chen https://orcid.org/0000-0002-8496-7870 


\section{REFERENCES}

1. Miszczuk GS, Banales JM, Zucchetti AE, Pisani GB, Boaglio AC, Saez E, et al. Adaptive downregulation of $\mathrm{Cl}$-/HCO3- exchange activity in rat hepatocytes under experimental obstructive cholestasis. PLoS One 2019;14:e0212215.

2. Wagner M, Trauner M. Transcriptional regulation of hepatobiliary transport systems in health and disease: implications for a rationale approach to the treatment of intrahepatic cholestasis. Ann Hepatol 2005;4:77-99.

3. Zollner G, Marschall HU, Wagner M, Trauner M. Role of nuclear receptors in the adaptive response to bile acids and cholestasis: pathogenetic and therapeutic considerations. Mol Pharm 2006;3: 231-51.

4. Wagner M, Zollner G, Trauner M. New molecular insights into the mechanisms of cholestasis. J Hepatol 2009;51:565-80.

5. Schuetz JD, Connelly MC, Sun D, Paibir SG, Flynn PM, Srinivas $\mathrm{RV}$, et al. MRP4: a previously unidentified factor in resistance to nucleoside-based antiviral drugs. Nat Med 1999;5:1048-51.

6. Borst P, Elferink RO. Mammalian ABC transporters in health and disease. Annu Rev Biochem 2002;71:537-92.

7. Wielinga PR, van der Heijden I, Reid G, Beijnen JH, Wijnholds J, Borst P. Characterization of the MRP4- and MRP5-mediated transport of cyclic nucleotides from intact cells. J Biol Chem 2003;278: 17664-71.

8. Schuetz EG, Strom S, Yasuda K, Lecureur V, Assem M, Brimer C, et al. Disrupted bile acid homeostasis reveals an unexpected interaction among nuclear hormone receptors, transporters, and cytochrome P450. J Biol Chem 2001;276:39411-8.

9. Wagner M, Fickert P, Zollner G, Fuchsbichler A, Silbert D, Tsybrovskyy $\mathrm{O}$, et al. Role of farnesoid $\mathrm{X}$ receptor in determining hepatic $\mathrm{ABC}$ transporter expression and liver injury in bile duct-ligated mice. Gastroenterology 2003;125:825-38.

10. Donner MG, Schumacher S, Warskulat U, Heinemann J, Häussinger D. Obstructive cholestasis induces TNF-alpha- and IL-1 -mediated periportal downregulation of Bsep and zonal regulation of Ntcp, Oatpla4, and Oatp1b2. Am J Physiol Gastrointest Liver Physiol 2007;293:G1134-46.

11. Stedman CA, Liddle C, Coulter SA, Sonoda J, Alvarez JG, Moore $\mathrm{DD}$, et al. Nuclear receptors constitutive androstane receptor and pregnane X receptor ameliorate cholestatic liver injury. Proc Natl Acad Sci U S A 2005;102:2063-8.

12. Petrick JS, Klaassen CD. Importance of hepatic induction of constitutive androstane receptor and other transcription factors that regulate xenobiotic metabolism and transport. Drug Metab Dispos 2007;35:1806-15.

13. Chen P, Zeng H, Wang Y, Fan X, Xu C, Deng R, et al. Low dose of oleanolic acid protects against lithocholic acid-induced cholestasis in mice: potential involvement of nuclear factor-E2-related factor 2-mediated upregulation of multidrug resistance-associated proteins. Drug Metab Dispos 2014;42:844-52.

14. Bohan A, Chen WS, Denson LA, Held MA, Boyer JL. Tumor necrosis factor alpha-dependent up-regulation of Lrh-1 and Mrp3 (Abcc3) reduces liver injury in obstructive cholestasis. J Biol Chem 2003;278:36688-98.

15. Chai J, He Y, Cai SY, Jiang Z, Wang H, Li Q, et al. Elevated hepatic multidrug resistance-associated protein 3/ATP-binding cassette subfamily C 3 expression in human obstructive cholestasis is mediated through tumor necrosis factor alpha and c-Jun NH2-terminal kinase/stress-activated protein kinase-signaling pathway.
Hepatology 2012;55:1485-94.

16. Zhang FX, Kirschning CJ, Mancinelli R, Xu XP, Jin Y, Faure E, et al. Bacterial lipopolysaccharide activates nuclear factor-kappaB through interleukin-1 signaling mediators in cultured human dermal endothelial cells and mononuclear phagocytes. J Biol Chem 1999;274:7611-4.

17. Lee J, Azzaroli F, Wang L, Soroka CJ, Gigliozzi A, Setchell KD, et al. Adaptive regulation of bile salt transporters in kidney and liver in obstructive cholestasis in the rat. Gastroenterology 2001;121: 1473-84.

18. Chai J, Luo D, Wu X, Wang H, He Y, Li Q, et al. Changes of organic anion transporter MRP4 and related nuclear receptors in human obstructive cholestasis. J Gastrointest Surg 2011;15:996-1004.

19. Renga B, Migliorati M, Mencarelli A, Cipriani S, D’Amore C, Distrutti E, et al. Farnesoid X receptor suppresses constitutive androstane receptor activity at the multidrug resistance protein-4 promoter. Biochim Biophys Acta 2011;1809:157-65.

20. Chai J, Cai SY, Liu X, Lian W, Chen S, Zhang L, et al. Canalicular membrane MRP2/ABCC2 internalization is determined by Ezrin Thr567 phosphorylation in human obstructive cholestasis. J Hepatol 2015;63:1440-8.

21. Denk GU, Soroka CJ, Takeyama Y, Chen WS, Schuetz JD, Boyer JL. Multidrug resistance-associated protein 4 is up-regulated in liver but down-regulated in kidney in obstructive cholestasis in the rat. J Hepatol 2004;40:585-91.

22. Geier A, Wagner M, Dietrich CG, Trauner M. Principles of hepatic organic anion transporter regulation during cholestasis, inflammation and liver regeneration. Biochim Biophys Acta 2007;1773: 283-308.

23. Osawa Y, Hoshi M, Yasuda I, Saibara T, Moriwaki H, Kozawa O. Tumor necrosis factor- $\alpha$ promotes cholestasis-induced liver fibrosis in the mouse through tissue inhibitor of metalloproteinase-1 production in hepatic stellate cells. PLoS One 2013;8:e65251.

24. Nagaki M, Naiki T, Brenner DA, Osawa Y, Imose M, Hayashi H, et al. Tumor necrosis factor alpha prevents tumor necrosis factor receptor-mediated mouse hepatocyte apoptosis, but not fas-mediated apoptosis: role of nuclear factor-kappaB. Hepatology 2000; 32:1272-9.

25. Malekshah OM, Lage H, Bahrami AR, Afshari JT, Behravan J. PXR and NF- $\kappa B$ correlate with the inducing effects of IL- $1 \beta$ and TNF- $\alpha$ on ABCG2 expression in breast cancer cell lines. Eur J Pharm Sci 2012;47:474-80.

26. Eba S, Hoshikawa Y, Moriguchi T, Mitsuishi Y, Satoh H, Ishida K, et al. The nuclear factor erythroid 2-related factor 2 activator oltipraz attenuates chronic hypoxia-induced cardiopulmonary alterations in mice. Am J Respir Cell Mol Biol 2013;49:324-33.

27. Trimarchi JM, Lees JA. Sibling rivalry in the E2F family. Nat Rev Mol Cell Biol 2002;3:11-20.

28. Wang S, Nath N, Minden A, Chellappan S. Regulation of Rb and E2F by signal transduction cascades: divergent effects of JNK1 and p38 kinases. EMBO J 1999;18:1559-70.

29. Whyte-Allman SK, Hoque MT, Jenabian MA, Routy JP, Bendayan R. Xenobiotic nuclear receptors pregnane $\mathrm{X}$ receptor and constitutive androstane receptor regulate antiretroviral drug efflux transporters at the blood-testis barrier. J Pharmacol Exp Ther 2017;363: 324-35.

30. Soroka CJ, Lee JM, Azzaroli F, Boyer JL. Cellular localization and up-regulation of multidrug resistance-associated protein 3 in hepatocytes and cholangiocytes during obstructive cholestasis in rat liver. Hepatology 2001;33:783-91. 